\title{
Avaliação Financeira do Manejo Florestal Comunitário
}

\section{Financial Assessment of Community Forest Management}

Gabriel Medina - Doutor em Ciências Florestais e Ambientais pela Universidade de Freiburg em 2008; professor da Universidade Federal de Goiás. E-mail: gabriel.silva.medina@gmail.com

Benno Pokorny - Doutor em Engenharia Florestal pela Universidade de Freiburg; professor da Universidade de Freiburg, Alemanha. E-mail: benno.pokorny@waldbau.uni-freiburg.de

\section{Resumo}

As comunidades da Amazônia têm grandes possibilidades de melhorar suas condições de vida, por meio do manejo de suas florestas. Existem diferentes iniciativas externas buscando apoiar as comunidades a estabelecerem sistemas de manejo florestal comunitário (MFC) para a extração madeireira. No entanto, não existe ainda uma avaliação da viabilidade financeira dos modelos propostos. Esse estudo, feito com oito iniciativas de MFC, revelou que a viabilidade financeira dos modelos atuais é bastante limitada. Um debate crítico sobre o potencial desses modelos técnicos externos precisa ser feito, buscando ajustar as propostas aos interesses e capacidades das comunidades.

\section{Palavras-chave}

Amazônia. Desenvolvimento rural. Extração de madeira. Comunidades rurais.

\begin{abstract}
Amazonian communities have the potential for improving their livelihoods by efficiently managing their forest resources. Aiming to support communities, there are different externally-driven initiatives promoting community forest management (CFM) for timber extraction. However, there is limited understanding of the financial viability of those initiatives. This study revealed that families received limited financial benefits from externally-driven CFM initiatives. A critical debate on the real potential of these approaches needs to take place in order to adjust the concepts to the interests and capacities of local communities.
\end{abstract}

\section{Keywords}

Amazon. Rural development. Timber extraction. Rural communities. 


\section{INTRODUÇÃO}

O Manejo Florestal Comunitário é assumido como um potencial para gerar renda e trabalho para os produtores familiares da Amazônia e contribuir para a manutenção das florestas. Sua promoção tem buscado a capacitação dos produtores em práticas de Exploração de Impacto Reduzido, organização em torno de cooperativas e associações e estratégias para a busca de mercados atrativos, incluindo a adoção de certificação. A partir da década de 1990, ONGs e agências governamentais, apoiados por doadores internacionais, começaram a estabelecer iniciativas-piloto como forma de demonstrar a viabilidade técnica e financeira do Manejo Florestal Comunitário (MEDINA et al., 2009). Adicionalmente, adaptações no marco legal e institucional foram feitas, como forma de regulamentar a atividade.

Após uma década de experiências com Manejo Florestal Comunitário, doadores e políticos estão em busca das lições aprendidas nas iniciativas-piloto, como base para a construção de políticas com maior abrangência. No entanto, ainda não existe uma avaliação sobre a viabilidade financeira dos diferentes modelos experimentados na região, como um aspecto crucial para avaliar seu real potencial de replicação. Os estudos existentes foram feitos em casos individuais (LIMA et al., 2006) ou analisaram os aspectos financeiros apenas superficialmente (SOUSA; GOMES, 2005; AMARAL; AMARAL, 2005). Esse trabalho consiste em uma primeira tentativa, neste sentido, promovida pelo Subprograma de Apoio ao Manejo Florestal Sustentável na Amazônia (ProManejo). O ProManejo é um programa executado pelo Ministério do Meio Ambiente, que promove a adoção de sistemas sustentáveis de manejo florestal na Amazônia, com ênfase em produtos madeireiros, por meio de ações estratégicas e projetos demonstrativos.

Esse trabalho traz uma avaliação financeira de iniciativas de manejo florestal comunitário apoiadas pelo ProManejo. A avaliação abordou os modelos técnicos adotados, os investimentos necessários para implementar estes modelos, as produtividades alcançadas, e as rentabilidades obtidas. Os resultados demonstraram que são modestas as possibilidades para os produtores gerarem benefícios financeiros nos modelos propostos. O estudo indicou a necessidade de uma revisão profunda no entendimento sobre o uso de florestas por produtores familiares, de forma a aproveitar as vantagens comparativas dos produtores familiares para o manejo florestal e desenvolvimento regional. 


\section{MATERIAL E MÉTODOS}

Foram analisadas oito iniciativas-piloto de manejo florestal comunitário apoiadas pelo ProManejo e apontadas como as mais promissoras e com melhor disponibilidade de informações documentadas. Nesta avaliação, estas iniciativas foram divididas em iniciativas de menor e maior escalas. Iniciativas em menor escala, como Oficinas Caboclas, Boa Vista dos Ramos, Mamirauá e Pedro Peixoto tiveram unidades de produção anual de até 50 ha, forma de trabalho coletiva e exploraram menos de $250 \mathrm{~m}^{3}$ por ano, de forma não mecanizada. As iniciativas em maior escala incluem Ambé, Costa Marques, Projetos de Assentamentos Agroextrativistas (PAE) Cachoeira e Equador, e Porto Dias, e tiveram áreas de produção anual superiores a 100 ha, com equipes de trabalho especializadas, e exploraram mais de $650 \mathrm{~m}^{3}$ por ano, de forma mecanizada (Tabela 1).

Em cada iniciativa, o levantamento de informações tomou três a quatro dias de trabalho de campo e envolveu entrevistas com os técnicos acompanhando as iniciativas, com os produtores e também visitas às áreas de exploração e processamento de madeira. Adicionalmente, foram revisadas as informações existentes nas organizações de apoio. A partir das especificidades observadas em cada iniciativa, definiu-se um modelo de manejo florestal a ser avaliado, considerando aspectos como as tecnologias e máquinas usadas, o tamanho da unidade de produção anual, o volume anualmente explorado, o valor dos investimentos e as receitas obtidas. Os custos com pessoal foram baseados no valor da diária paga ou, em caso de iniciativas sem remuneração direta, no valor da diária local. Os custos com máquinas consideraram, além do consumo, os custos de capital, depreciação e manutenção. Os custos de capital foram estimados com juros de 5\%, valor cobrado pelo Banco da Amazônia em créditos para pequenos empreendimentos. A depreciação foi calculada em forma linear para um período de 10 anos e os custos com manutenção foram definidos como $60 \%$ do valor da depreciação. 


\begin{tabular}{|c|c|c|c|c|c|c|}
\hline \multicolumn{2}{|c|}{ 号: } & 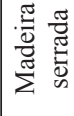 & 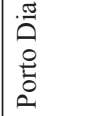 & 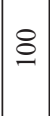 & ஜூ & $\begin{array}{l}0 \\
\infty \\
\infty \\
0 \\
0 \\
d\end{array}$ \\
\hline \multicolumn{2}{|c|}{ 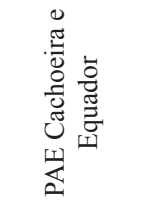 } & $\begin{array}{l}\frac{\pi}{0} \\
\frac{\pi}{0} \\
0 \\
0 \\
\frac{\pi}{0} \\
\frac{\pi}{0} \\
\sum\end{array}$ & 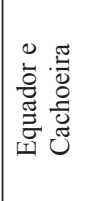 & in & 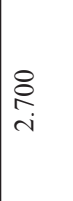 & ర్ \\
\hline \multicolumn{2}{|c|}{ 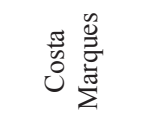 } & 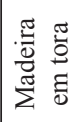 & 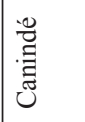 & $\stackrel{8}{\circ}$ & $\begin{array}{l}\stackrel{\infty}{\infty} \\
\stackrel{\sim}{+}\end{array}$ & ' \\
\hline \multicolumn{2}{|c|}{ 娄 } & 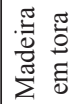 & 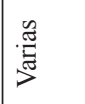 & $\stackrel{8}{8}$ & $\frac{\partial}{\partial}$ & ' \\
\hline \multirow{2}{*}{$\begin{array}{l}0 \\
0 \\
.0 \\
0 \\
0 \\
0 \\
0 \\
0 \\
0 \\
0\end{array}$} & \multirow{2}{*}{ 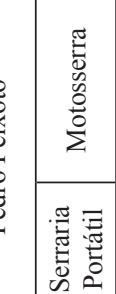 } & \multirow{2}{*}{ 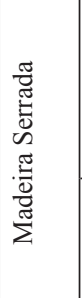 } & $\begin{array}{l}0 \\
0 \\
.0 \\
0 \\
0 \\
2 \\
0 \\
0 \\
0 \\
0 \\
2\end{array}$ & $\nabla$ & $\stackrel{\infty}{\stackrel{\infty}{0}}$ & $\begin{array}{l}\vec{\sigma} \\
\vec{\sigma}\end{array}$ \\
\hline & & & 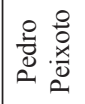 & + & $\stackrel{\infty}{\stackrel{\infty}{=}}$ & $\begin{array}{l}\text { D } \\
\text { in }\end{array}$ \\
\hline \multirow{3}{*}{ 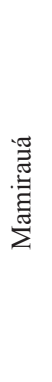 } & 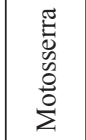 & \multirow{2}{*}{ 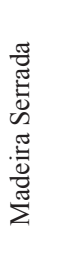 } & 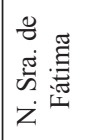 & 은 & 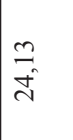 & $\begin{array}{l}+ \\
\infty \\
0 \\
\varrho\end{array}$ \\
\hline & 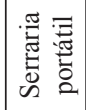 & & 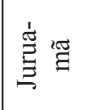 & $\circ$ & 总 & $\begin{array}{l}n \\
\text { ñ } \\
\stackrel{n}{n}\end{array}$ \\
\hline & 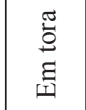 & 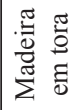 & 学㺃 & त & הి & ' \\
\hline \multicolumn{2}{|c|}{ 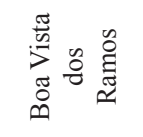 } & 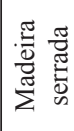 & 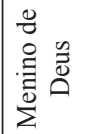 & q & $\underset{\infty}{\stackrel{\sim}{\infty}}$ & P \\
\hline \multirow{2}{*}{ 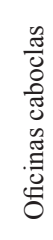 } & $\begin{array}{l}\frac{\pi}{\pi} \frac{\pi}{\pi} \\
\frac{\pi}{\pi} \\
\Sigma\end{array}$ & \multirow{2}{*}{ 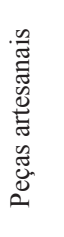 } & \multirow{2}{*}{ 夆 } & - & $\begin{array}{l}n \\
n \\
n\end{array}$ & 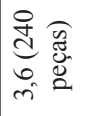 \\
\hline & 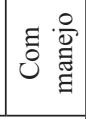 & & & q & $\begin{array}{l}\tilde{n} \\
i n\end{array}$ & 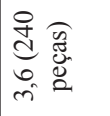 \\
\hline & & $\begin{array}{l}\stackrel{0}{3} \\
\frac{3}{0} \\
\text { : }\end{array}$ & 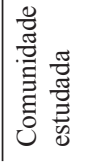 & 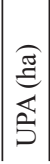 & 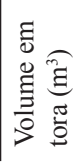 & 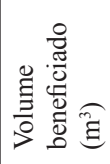 \\
\hline
\end{tabular}




\section{RESULTADOS E DISCUSSÃO}

\subsection{Investimentos}

O Manejo Florestal Comunitário parte do princípio de que os produtores têm a capacidade de manejar suas florestas de acordo com as técnicas de Exploração de Impacto Reduzido, inicialmente desenvolvidas para empresas. Nas iniciativas estudadas, quanto maior a escala, maior foi a semelhança com as operações realizadas por empresas. No entanto, o estudo mostrou que os produtores não atuaram em todas as atividades. Eles desempenharam, com relativa autonomia, as atividades como delimitação da área, inventário, derruba e serragem. Outras atividades, no entanto, permaneceram realizadas por engenheiros ou técnicos florestais, ou foram terceirizadas. A preparação do plano de manejo e o planejamento das atividades de campo foram feitos principalmente pela equipe de acompanhamento técnico, enquanto as atividades que envolviam o uso de máquinas pesadas foram, em geral, terceirizadas.

O conceito de Manejo Florestal Comunitário sugere que, uma vez que é dado um pequeno apoio inicial, depois de um tempo de consolidação, os produtores podem continuar manejando por conta própria. A avaliação revelou que foram feitos investimentos iniciais significativamente altos pelas organizações de apoio. Especificamente foram necessários altos investimentos na capacitação dos produtores para manejarem suas florestas, de acordo com o marco legal e os modelos técnicos propostos. Em geral, quanto maior a complexidade do arranjo produtivo (maior número de etapas como beneficiamento, busca de mercados externos etc.) maiores foram os custos com capacitação. Também, a aquisição dos equipamentos e máquinas para as operações do campo, em particular para o transporte e o beneficiamento da produção, representou custos significativos. No total, os investimentos iniciais variaram de $\mathrm{R} \$ 40$ mil a $\mathrm{R} \$ 1$,6milhão (Figura 1). 
Figura 1. Principais formas de investimentos feitos nas iniciativas estudadas (R\$).

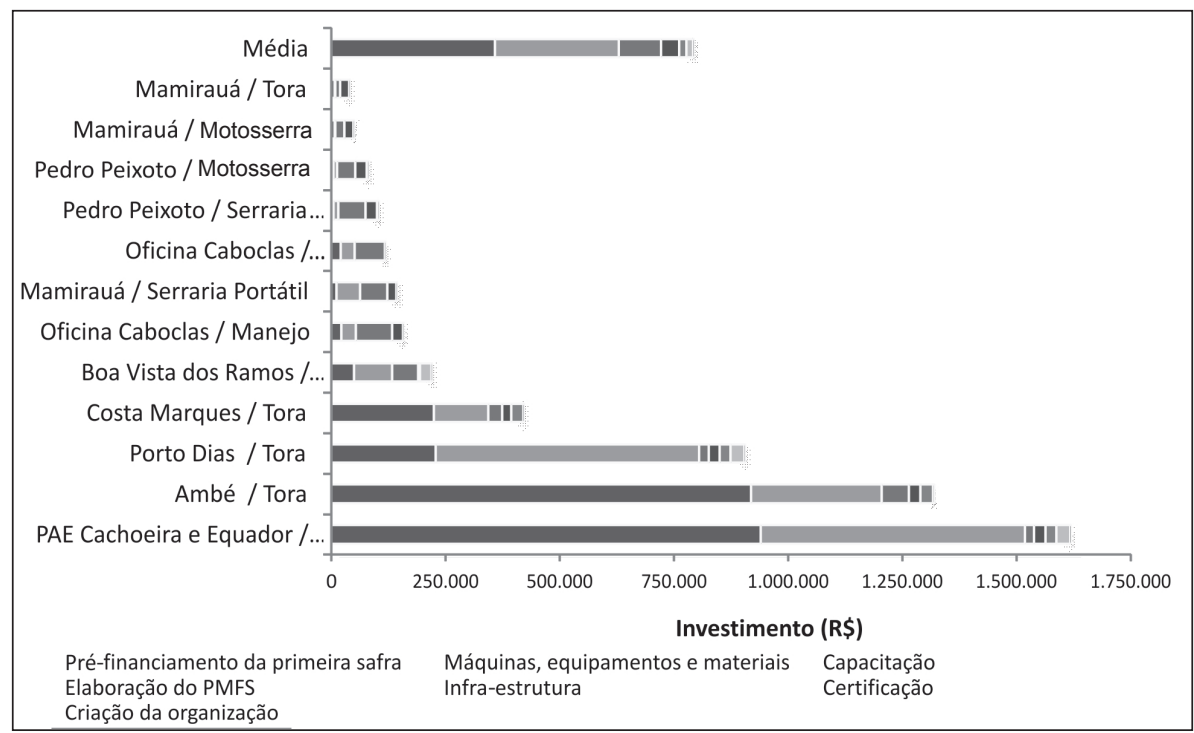

Fonte: Pesquisa de campo.

\subsection{Produtividade}

As iniciativas foram concebidas com a expectativa de trabalhar com produtividades e custos competitivos. Em relação às produtividades, o estudo observou um gradiente que vai das iniciativas em menor escala com produtividades $75 \%$ menores que empresas para as iniciativas em maior escala produtividades $25 \%$ menores que empresas (Tabela 2). Aparentemente, para as iniciativas em maior escala, o pacote tecnológico se mostrou mais adequado.

As produtividades relativamente baixas nas iniciativas estudadas, resultaram, além do efeito da escala, das próprias características do modo de produção familiar, incluindo: o interesse em maximizar a remuneração da mão de obra e não o lucro, a valorização do tempo livre (folga, intervalos) e a importância de outras atividades produtivas (como agricultura). Também, as pressões externas foram menores que nas empresas. As iniciativas operaram a partir de estruturas mais horizontais e tiveram seus custeios iniciais (incluindo a compra de máquinas e investimentos iniciais) pagos pelo ProManejo. 
Tabela 2. Produtividades nos diferentes tipos de manejo.

\begin{tabular}{l|c|c|c|c}
\hline \multirow{2}{*}{} & \multicolumn{2}{|c|}{ Menor escala } & \multicolumn{2}{c}{ Maior escala } \\
\cline { 2 - 5 } & Mini & Pequeno & Grande & Empresas \\
\cline { 2 - 5 } & $\begin{array}{c}\text { Oficinas } \\
\text { Caboclas }\end{array}$ & $\begin{array}{c}\text { Mamirauá, Pedro } \\
\text { Peixoto, BVR }\end{array}$ & $\begin{array}{c}\text { Ambé, PAE, } \\
\text { Porto Dias, Corta } \\
\text { Marques }\end{array}$ & Cikel, Juruá, IBL \\
\hline Delimitação (ha por dia) & 2 & 3 & 9 & 18 \\
\hline Inventário (ha por dia) & 2 & 3 & 11 & 12 \\
\hline Derrubada (m por dia) & 0,5 & 14 & 40 & 55 \\
\hline Arraste $\left(\mathrm{m}^{3}\right.$ por dia) & 0,5 & 3 & 59 & 75 \\
\hline
\end{tabular}

Fonte: Pesquisa de campo.

\subsection{Custos}

Nas iniciativas avaliadas, os custos de produção, como consequência das produtividades relativamente baixas, foram significativamente altos, quando comparados com empresas. A produção de um metro cúbico em tora gerou custos entre $\mathrm{R} \$ 29,00$ e $\mathrm{R} \$ 100,00$. Os custos para produzir um metro cúbico serrado com motosserra ficaram na faixa de $\mathrm{R} \$ 690,00$ a $\mathrm{R} \$ 840,00$. Quando serrado com serraria portátil, os custos variaram de $\mathrm{R} \$ 370,00$ a $\mathrm{R} \$ 1.235,00$ por metro cúbico, influenciados pelo maior ou menor aproveitamento da capacidade da máquina. As iniciativas que terceirizaram a serragem da madeira tiveram custos finais aproximados de $\mathrm{R} \$ 845,00$ por metro cúbico serrado. Nas oficinas caboclas, como exemplo de uma iniciativa verticalizada até o produto final, os custos médios de produção variaram de $\mathrm{R} \$$ 84,00 (com madeiras caídas) a $\mathrm{R} \$$ 96,00 (com manejo) por cada peça (Tabela 3).

Nas iniciativas em menor escala, destacaram-se os custos de acompanhamento técnico e custos administrativos. Nas Oficinas Caboclas, por exemplo, os principais custos foram com a manutenção da cooperativa criada para vender a produção para mercados externos. Na iniciativa de exploração de madeira em tora em Mamirauá, o acompanhamento técnico representou o maior custo. Nas iniciativas de serragem com motosserra e serraria portátil, os custos das máquinas foram os mais significativos. Nestas iniciativas, os custos com impostos incidindo sobre o valor de venda da madeira processada também foram relevantes. Nas iniciativas em maior escala, destacaram-se os custos com as máquinas na construção de infraestrutura, arraste e carregamento. Nas iniciativas que beneficiaram a madeira, além dos custos com as máquinas, destacaram-se os custos com administração, para conseguir vender a madeira serrada para mercados externos. 


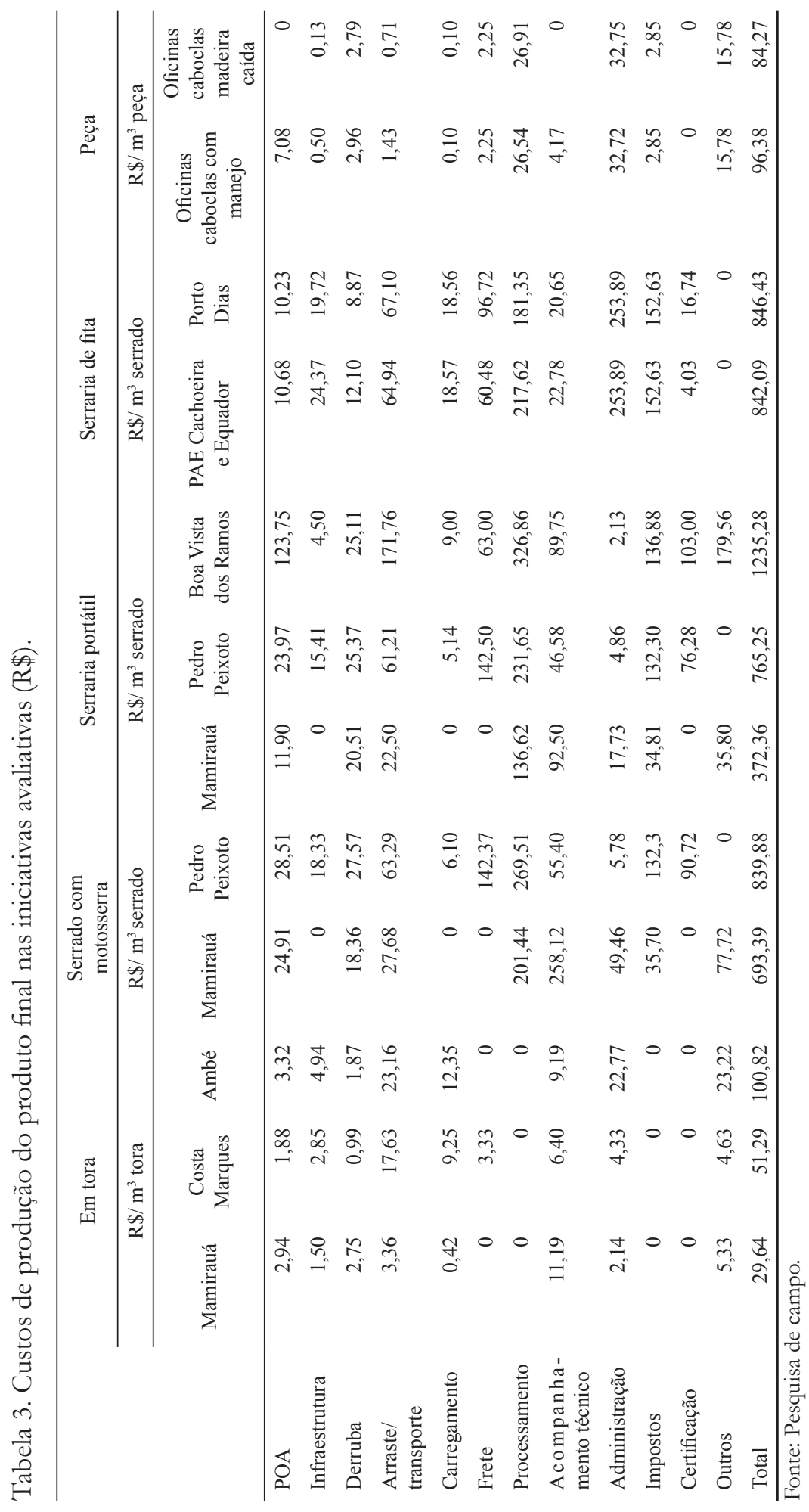




\subsection{Rentabilidade}

O conceito do Manejo Florestal Comunitário partiu do princípio de que é uma atividade financeiramente interessante para o produtor familiar e representa baixo risco. A análise revelou, porém, que os rendimentos são modestos e os riscos são relativamente altos. Das iniciativas estudadas, apenas aquelas em maior escala e com menor verticalização do arranjo produtivo conseguiram remunerar a mão de obra e ainda gerar receita líquida (Ambé, Costa Marques e Mamirauá). Outras iniciativas conseguiram ter um resultado financeiramente positivo se não são consideradas as diárias pagas, indicando que podem remunerar a mão de obra abaixo dos valores atualmente pagos (Oficinas Caboclas, Pedro Peixoto, Porto Dias e PAE). Porém, outras iniciativas nem conseguiram cobrir os custos operacionais sem subsídios externos, mesmo considerando que vendem para mercados que pagam preços superiores ao mercado local (BVR, Mamirauá, com serraria portátil e motosserra, e Pedro Peixoto, com motosserra) (Figura 3).

Também, o pressuposto de que a verticalização da produção agrega valor ao produto vendido e aumenta a receita final não se confirmou. A verticalização aumentou o valor de venda do produto final, mas também aumentou os custos de produção ainda em maior proporção. As iniciativas verticalizadas, pela maior complexidade do arranjo produtivo e maiores custos de produção, demandaram maiores capacidades administrativas. Iniciativas que buscaram atingir mercados externos tiveram custos administrativos significativos. Iniciativas que adotaram a certificação do sistema de manejo tiveram custos altos de adesão, auditorias e anuidades. A verticalização implicou, também, na maior participação dos custos fixos (máquinas), maior custo de pré-financiamento da safra, provocando maior risco e, consequentemente, menor flexibilidade.

Finalmente, o estudo mostrou a necessidade de investimentos contínuos para assegurar a manutenção das iniciativas. Nas iniciativas em menor escala, o capital de giro anual necessário variou de $\mathrm{R} \$ 7,5$ mil até $\mathrm{R} \$ 50$ mil. Nas iniciativas em maior escala, o capital de giro variou de $\mathrm{R} \$ 220$ mil até $\mathrm{R} \$ 940$ mil. A necessidade de capital de giro demanda a capacidade gerencial de assegurar recursos para financiar a próxima safra e rendimentos suficientes na safra anterior para gerar o capital de giro para a próxima safra. Como parte significativa das iniciativas ainda prescinde da capacidade gerencial necessária para garantir a manutenção do capital de giro, é previsível certa dificuldade para conquistarem autonomia financeira. As iniciativas avaliadas estão atualmente enfrentando este desafio com o fim do apoio do ProManejo. 
Figura 2. Receita líquida por produto final ( $\mathrm{R} \$)$.

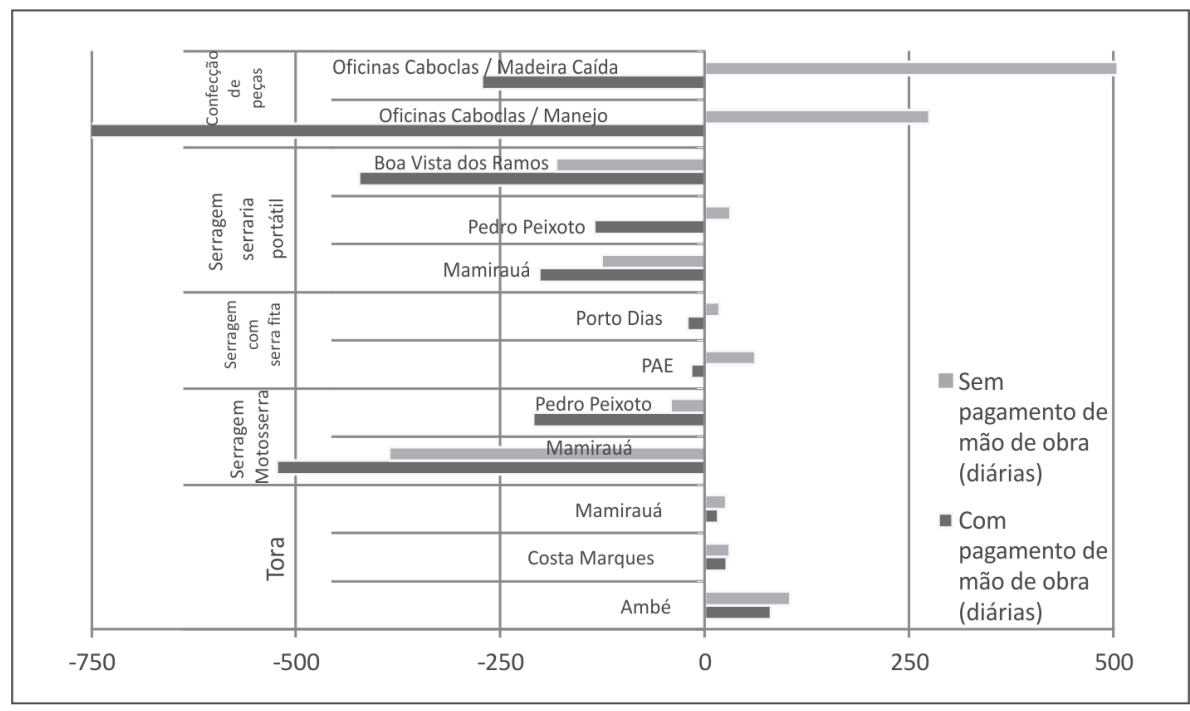

Fonte: Pesquisa de campo.

\section{CONCLUSÕES}

O estudo das iniciativas de manejo florestal comunitário na Amazônia mostrou a necessidade de avaliar criticamente a viabilidade dos modelos implementados atualmente. Os modelos estudados têm rentabilidade financeira limitada, exigem altos investimentos na implementação e tendem a demandar subsídios constantes. Porém, na avaliação, é indispensável distinguir entre iniciativas em menores e maiores escalas.

Em iniciativas em menor escala, o uso eficiente da madeira representa uma oportunidade importante para produtores familiares terem um complemento de renda. Porém, os modelos adotados implicaram em custos relativamente altos com o acompanhamento técnico e a administração de estruturas verticalizadas. Para viabilizar estas iniciativas, as práticas de manejo e os arranjos produtivos precisam ser adaptados às necessidades e capacidades dos produtores. Algumas práticas inspiradas no manejo empresarial são facilmente substituíveis por práticas mais simples e com menor dependência de tecnologia e acompanhamento externo. A simplificação de outras práticas, como a obrigatoriedade da anuência de um engenheiro florestal, demanda a revisão do marco legal. A verticalização e a certificação devem ser adotadas somente quando significarem benefícios reais. Também há situações em que o manejo florestal é simplesmente uma atividade 
dispensável para o produtor familiar, que tem outras prioridades. Neste sentido, o enfoque em madeira pode entrar em conflito com o enfoque tradicional do produtor familiar na diversidade de produtos extrativos.

As iniciativas em maior escala representam uma opção para o manejo de grandes áreas de florestas de comunidades, assim como de florestas públicas, por produtores familiares organizados. Diferente da exploração por empresas, o manejo por produtores familiares tem a característica de uma maior distribuição da renda gerada entre os associados. Adicionalmente, o regime de trabalho mais flexível é mais compatível com as tradições dos comunitários.

Entretanto, estabelecer iniciativas em maior escala implica etapas fundamentais, como a criação de uma representação legal para administrar a iniciativa; a contratação de uma equipe de apoio para atividades específicas, como administração financeira e acompanhamento técnico; a capacitação dos manejadores; e a possível terceirização do arraste e transporte que demandam grandes inversões em maquinários. Isso implica investimentos inicias consideráveis, que demandam linhas de créditos específicas e apoio governamental. O estabelecimento destes arranjos produtivos demanda que o Estado privilegie e apoie o manejo florestal feito pelos próprios produtores, por exemplo, por meio de apoio na concessão de florestas públicas, oferta de créditos e redução dos impostos. Considerando a competição potencial com uma empresa manejando florestas públicas, a sociedade tem que fazer uma escolha: receber as taxas das concessões feitas a empresas ou contribuir diretamente para o desenvolvimento local apoiando iniciativas por produtores familiares.

\section{AGRADECIMENTOS}

Este estudo foi elaborado com base em uma pesquisa para avaliar oito iniciativas-piloto de manejo florestal comunitário na Amazônia Brasileira apoiadas pelo Programa de Apoio ao Manejo Florestal Sustentável na Amazônia (ProManejo) implementado pelo Ministério do Meio Ambiente (MMA), por meio do Instituto Brasileiro do Meio Ambiente e dos Recursos Naturais Renováveis (Ibama). Os nossos mais sinceros agradecimentos à equipe do ProManejo, em particular a Ricado Ludke e às comunidades que nos receberam durante a pesquisa de campo. 


\section{REFERÊNCIAS}

AMARAL, P.; AMARAL, M. Manejo florestal comunitário: processos e aprendizagens na Amazônia brasileira e na América Latina. Belém: IEB; Imazon, 2005.

LIMA, E.; MERRY, F.; NEPSTAD, D.; AMACHER, G.; RAMOS, C.; LEFEBVRE, P.; RESQUE, F. Searching for sustainability: forest policies, smallholders and the Trans-amazon highway. Environment, v. 48, n.1, p. 26-38, 2006.

MEDINA, G.; POKORNY, B.; CAMPBELL, B. Community forest management for timber extraction in the Amazon frontier. International Forestry Review, v. 11, p. 408-420, 2009.

SOUSA, R.; GOMES, D. Produção familiar rural: tendências e oportunidades da atividade madeireira no Acre e Pará. Belém: GTNA; Forest Trends; IEB, 2005. 